\title{
MODELACIÓN DE LA DISPERSIÓN DE ANHÍDRIDO SULFUROSO EN LA COMUNA DE PUCHUNCAVÍ UTILIZANDO EL PROGRAMA ISC3
}

\section{MODELING OF THE SULFUR DIOXIDE DISPERSION IN THE PUCHUNCAVI CITY USING THE PROGRAM ISC3}

\author{
Pamela Lazo ${ }^{1} \quad$ Michel Curé ${ }^{2} \quad$ Hernán Gaete $^{3}$ \\ Recibido 13 de marzo de 2006, aceptado el 26 de septiembre de 2006 \\ Received: March 13, 2006 Accepted: September 26, 2006
}

\begin{abstract}
RESUMEN
El presente trabajo evalúa el modelo de dispersión atmosférica ISC3 en la comuna de Puchuncaví, modelando las emisiones de $\mathrm{SO}_{2}$ provenientes del Complejo Industrial Las Ventanas en las cinco estaciones que conforman la red de calidad del aire de este complejo. Se modelan concentraciones horarias y promedio de 24 horas para los años 2003 y 2004, minimizando los errores sistemáticos mediante la aplicación del filtro Kalman. Se evalúa el desempeño del modelo mediante una serie de herramientas estadísticas. Se realiza un análisis de sensibilidad de la variación en la tasa de emisión de $\mathrm{SO}_{2}$, velocidad y dirección del viento, observando que el modelo es sensible a estos factores y siendo una de las causas de los errores obtenidos en cuatro de las cinco estaciones de calidad del aire del complejo industrial. Se concluye que el modelo ISC3 predice con menor error en la dirección del viento predominante en frecuencia, entregando un mejor ajuste para velocidades de viento elevadas en direcciones de viento menores en frecuencia. Se observa que el filtro de Kalman mejora el desempeño del modelo ISC3 entregando resultados con menor error en las cinco estaciones de calidad del aire.
\end{abstract}

Palabras clave: Contaminación atmosférica, modelación, dióxido de azufre, dispersión, filtro de Kalman.

\begin{abstract}
The present work evaluates the atmospheric dispersion model ISC3 in Puchuncavi, Chile, modelling the emissions of $\mathrm{SO}_{2}$ coming from the five stations that make up the air quality network in the industrial complex of Ventanas. 24 hour average concentrations for the years 2003 and 2004 were modelled, systematic errors being diminished by using a Kalman filter. The performance of the model is evaluated using a series of statistic tools. An analysis of the sensitivity of the $\mathrm{SO}_{2}$ rate of emission, speed and wind direction variation is made, noting that the model is sensible to these factors, being this one of the causes of the errors found in four of the five stations that measured the quality of the air. It is concluded that the Kalman filter improves model ISC3, providing results with fewer errors in the five air quality measuring stations.
\end{abstract}

Keywords: Atmospheric, pollution, modeling, sulfur dioxide, dispersion, Kalman filter.

\section{INTRODUCCIÓN}

Debido a los cambios en el medio ambiente producidos por la actividad humana, ha sido necesario desarrollar herramientas que permitan predecir y evaluar estos cambios, y éstas son los modelos de dispersión. El empleo de modelos de dispersión atmosférica permite estimar las concentraciones totales de un contaminante en estudio, como también identificar las causas de niveles extraordinarios de contaminación. Es por esto que una serie de organismos internacionales se han dedicado a la tarea de impulsar el uso de este tipo de herramientas. De este modo, a partir de 1970 la Agencia de Protección Ambiental de los Estados Unidos (EPA) ha venido desarrollando una serie de programas regulatorios para la modelización de la calidad del aire; uno de estos programas es el ISC en su tercera versión, desarrollado por Pacific Environmental Services, Inc.

\footnotetext{
1 Ingeniería Ambiental, Facultad de Ciencias Universidad de Valparaíso, Chile.

2 Departamento de Física y Astronomía, Facultad de Ciencias, Universidad de Valparaíso, Valparaíso-Chile, michel.cure@uv.cl

3 Departamento de Biología y Ciencias Ambientales, Facultad de Ciencias, Universidad de Valparaíso, Valparaíso-Chile.
} 
En Chile, las actividades de fundición y refinación de cobre como de generación energética constituyen una fuente importante de contaminación atmosférica, debido a que sus procesos generan grandes cantidades de anhídrido sulfuroso. Así en la V Región encontramos la comuna de Puchuncaví que ha sido por años una zona fuertemente afectada por las emisiones de anhídrido sulfuroso provenientes del Complejo Industrial Las Ventanas, compuesto por Fundición y Refinería Las Ventanas y termoeléctrica AES Gener S.A. [1].

El presente trabajo tiene por objetivo validar el modelo de dispersión atmosférica ISC3, mediante la modelación de anhídrido sulfuroso en la comuna de Puchuncaví, considerando las emisiones provenientes del Complejo Industrial Las Ventanas. Se modelan concentraciones horarias y promedio de 24 horas de anhídrido sulfuroso para los años 2003 y 2004 en las cinco estaciones que conforman la red de calidad del aire de este complejo industrial, se minimizan los errores sistemáticos de los resultados del modelo ISC3 mediante la aplicación del filtro de Kalman y finalmente se realiza un análisis estadístico de las concentraciones modeladas y filtradas.

\section{ANTECEDENTES GENERALES}

\section{Antecedentes de las fuentes emisoras}

En la comuna de Puchuncaví se encuentra ubicado el Complejo Industrial Las Ventanas, compuesto por la Fundición y Refinería Las Ventanas perteneciente a CODELCO y por la Planta Termoeléctrica AES Gener S.A. Durante años la comuna de Puchuncaví ha sido fuertemente impactada por la operación de este complejo industrial, por ello el Ministerio de Minería a través del D.S. Nº185/1991 [2] obligó al Complejo Industrial Las Ventanas a instalar una red de monitoreo continuo de calidad del aire y a presentar un Plan de descontaminación a más tardar el 31 de julio de 1992.

El proyecto de red de monitoreo fue aprobado el 29 de abril de 1992, a su vez, el Plan de descontaminación fue aprobado mediante D.S. N 252 el 30 de diciembre de 1992 y en este último se estableció la obligación del Complejo Industrial Las Ventanas a cumplir las normas de calidad del aire para anhídrido sulfuroso a más tardar el 30 de junio de 1999, como también un cronograma de reducción de emisiones anuales de azufre como elemento formador de anhídrido sulfuroso y material particulado respirable; posteriormente y debido a las excedencias a las normas de calidad del aire de las mediciones entregadas por la red de monitoreo, el 9 de diciembre de 1993 mediante D.S. $\mathrm{N}^{\mathrm{o}} 346$ se declara zona saturada por anhídrido sulfuroso y material particulado el área jurisdiccional correspondiente a las comunas de Puchuncaví y Quintero.

En consecuencia, el tiempo de residencia del anhídrido sulfuroso es de unas pocas semanas ya que rápidamente se oxida a ácido sulfúrico, así, a pesar de la elevada velocidad de emisión, tanto por medios naturales como antropogénicos, su concentración en la troposfera es baja [3].

\section{El modelo utilizado}

El programa utilizado corresponde al modelo de fuente industrial compleja ISC, el cual incluye un modelo de largo plazo (ISCLT) y otro de corto plazo (ISCST), este último utilizado en el presente trabajo. El modelo ISC3 ha sido originalmente desarrollado para MS-DOS y se encuentra disponible en Internet en www.epa.gov/ scram001/tt22.htm

El modelo ISC de corto plazo se basa en la línea recta y ecuación gaussiana de la pluma en estado estacionario, y permite modelar un amplio rango de fuentes (puntuales, de volumen, de área y de foso abierto) estimando el valor de concentración o deposición para cada fuente y combinación de receptores para cada hora de entrada meteorológica, calculando los promedios de corto plazo especificados por el usuario [5], [6].

El modelo ISCST3 para chimeneas usa la ecuación gaussiana de la pluma en estado estacionario. La concentración horaria a una distancia $\mathrm{x}$ (en metros) en dirección del viento y a una distancia transversal y (en metros) está dada por:

$$
\chi=\frac{Q \cdot K \cdot V \cdot D}{2 \pi \cdot u_{S} \cdot \sigma_{y} \cdot \sigma_{z}} \cdot \exp \left[-0.5 \cdot\left(\frac{y}{\sigma_{y}}\right)^{2}\right]
$$

Donde Q es la tasa de emisión del contaminante, $\mathrm{K}$ es el coeficiente de escalamiento para convertir $[\mathrm{g} / \mathrm{s}]$ en $\left[\mu \mathrm{g} / \mathrm{m}^{3}\right]$, $\mathrm{V}$ es el término vertical, $\mathrm{D}$ es el término de decaimiento, $\sigma_{\mathrm{y}}, \sigma_{\mathrm{z}}$ son las desviaciones estándar de distribución de concentración lateral y vertical $\mathrm{y}_{\mathrm{s}}$ es la velocidad media del viento a la altura de la chimenea en $[\mathrm{m} / \mathrm{s}]$.

El modelo ISCST3 requiere dos archivos básicos: el archivo de datos de entrada de flujos y el archivo de datos meteorológicos. El archivo de datos de entrada de flujos está dividido en seis rutas: $\mathrm{CO}$ - Para especificar las opciones de control, SO - Para especificar las opciones 
de fuente, RE - Para especificar las opciones de receptor, $\mathrm{ME}$ - Para especificar la información meteorológica, TG - Para especificar la información de la grilla de terreno y OU - Para especificar las opciones de fuente, donde sólo la ruta TG es opcional. En el presente trabajo, además de obtener las concentraciones predichas por un modelo de calidad del aire, se minimiza el error sistemático de los resultados entregados a través de la utilización de una herramienta numérica como es el filtro de Kalman

\section{Algoritmo del filtro de Kalman}

El filtro de Kalman es un algoritmo matemático, que constituye el principal procedimiento para estimar sistemas dinámicos, entregando una solución recursiva y óptima al problema de filtrado lineal de datos discretos mediante el método de mínimos cuadrados. El filtro opera mediante el mecanismo de predicción y corrección, pronosticando un nuevo estado a partir de una estimación previa de éste, por medio de un término de corrección proporcional al error de predicción, lo cual lleva a la minimización de este último. [7]

El objetivo del filtro consiste en calcular un estimador lineal, insesgado y óptimo, que permita la actualización de información del estado de un sistema en un momento “t”, basándose en información disponible en " $t-1$ ".

Cuando se desea estimar una constante " $m$ " se realizan muchas medidas de ella. Sea $y$ una de las medidas que se realizan, tal como se expresa en la ecuación 2 , donde el ruido de la medición es $v$, con media cero y varianza $\sigma_{v}^{2}[8]$.

$$
y=m+v
$$

Sea $x_{0}$ una primera estimación de "m" con ruido w y varianza $\sigma_{w}^{2}$, como se muestra a continuación:

$$
x_{0}=m+w
$$

Al realizar una nueva estimación de $\mathrm{m}$, llamada $x_{1}$, su forma viene dada por:

$$
x_{1}=x_{0}+k\left(y-x_{0}\right)
$$

Donde $k$ recibe el nombre de ganancia Kalman y se obtiene a partir de los ruidos de las dos primeras estimaciones, tal como se muestra en el ecuación 5 .

$$
k=\frac{\sigma_{w}^{2}}{\sigma_{w}^{2}+\sigma_{v}^{2}}
$$

Así, esta nueva estimación es función lineal de las dos estimaciones anteriores, además es insesgada y óptima, y logra minimizar estadísticamente el error.

\section{Zona de estudio}

La comuna de Puchuncaví posee una superficie aproximada de $300 \mathrm{~km}^{2}$, cuenta con una población cercana a los 12.954 habitantes con un crecimiento del $21,5 \%$ con respecto al censo de 1992 . Las actividades principales son la pesca artesanal, comercio, turismo y actividades industriales [1], [9].

El valle de Puchuncaví es una zona de suelos pobres, ya que las deposiciones ácidas producto de la actividad industrial han destruido la vegetación acelerando el proceso de erosión, por lo cual el valle ha perdido fertilidad llevando al cese de los cultivos de cereales tradicionales en la zona. El clima presenta las mismas características de Chile Central, con veranos calurosos al interior y de menor intensidad en el sector costero.

Las fuentes emisoras de anhídrido sulfuroso bajo estudio de Fundición y Refinería Las Ventanas y AES Gener se encuentran ubicadas entre las coordenadas 267000 y 268000 UTM-E [m] y 6370000 y 6380000 UTM-N [m] [10].

Fundición y Refinería Las Ventanas y AES Gener cuentan con una red de monitoreo, la cual está conformada por seis estaciones meteorológicas, cinco de las cuales conforman la red de calidad del aire [11]. De acuerdo al análisis del régimen de viento, realizado a partir de los datos meteorológicos registrados en la estación meteorológica Principal y obtenidos en CONAMA, se observa -para los 23 meses analizados dentro del periodo correspondiente a los años 2003 y 2004-que la dirección del viento predominante en frecuencia para el periodo diurno correspondió a la oeste y oeste-noroeste, en cambio en el periodo nocturno correspondió a la dirección este.

\section{METODOLOGÍA}

Para el logro de los objetivos planteados se realizaron una serie de etapas metodológicas, las cuales se presentan a continuación.

\section{Recopilación y análisis de información}

La información meteorológica correspondiente a la estación Principal, así como las mediciones de concentración de anhídrido sulfuroso de la red de calidad del aire del 
Complejo Industrial Las Ventanas se obtuvieron en CONAMA, a su vez, la información de caracterización de las fuentes consideradas se obtuvo del documento Evaluación del cumplimiento del Plan de descontaminación de Ventanas [11].

Se trabajó con las tasas de emisión correspondientes al promedio anual, por lo que durante el periodo de ejecución del modelo se asumió una operación constante.

\section{Ejecución del modelo ISC3}

Para la ejecución del modelo ISC3 se crearon dos tipos de archivos básicos, ambos en formato ASCII. El primero de ellos correspondiente al archivo meteorológico consideró las mediciones horarias provenientes de la estación Principal relativas a los parámetros de dirección y velocidad del viento, temperatura, altura de la capa de mezcla y clase de estabilidad atmosférica. El segundo archivo requerido correspondiente al archivo de entrada de flujos comprendió información referente al periodo de promediación, el contaminante modelado, el tipo de terreno, los parámetros de las fuentes consideradas, los receptores considerados y el tipo de información de salida.

\section{Condiciones y periodo a modelar}

Se definió el periodo de ejecución del presente trabajo como enero-diciembre 2003 y enero-noviembre 2004.
Se realizó la modelación de concentraciones horarias y promedio de 24 horas de anhídrido sulfuroso para cada mes dentro del periodo considerado. La modelación se efectuó para cada una de las cinco estaciones de calidad del aire del Complejo Industrial Las Ventanas, cuya localización se presenta en la tabla 1 . Así también en la tabla 2 se presenta la localización de las fuentes emisoras consideradas.

\section{Manejo de los archivos de salida y análisis de los resultados}

Los archivos resultantes de la ejecución del modelo ISC3 se manejaron mediante el lenguaje de programación Perl (Practical extraction and report language) [12], [13]. Se analizaron los resultados correspondientes a las concentraciones horarias para cada mes, como también los promedios diarios para cada día dentro del periodo a modelar.

A los resultados obtenidos de la modelación se les aplicó el filtro Kalman [8] con la finalidad de minimizar los errores sistemáticos del modelo. Para las concentraciones horarias modeladas se aplicó el filtro por mes, utilizando las 14 primeras horas de cada mes para la estabilización del filtro. Para las concentraciones promedio de 24 horas se aplicó el filtro por año, utilizando los 14 primeros días de enero para estabilizar el filtro en el período 2003 y los 14 últimos días del mes de diciembre del 2003 para estabilizar el filtro para el año 2004.

Tabla 1. Estaciones de calidad del aire Complejo Industrial Las Ventanas.

\begin{tabular}{|c|c|c|c|}
\hline Estación de Monitoreo & $\begin{array}{c}\text { UTM-E } \\
{[\mathrm{m}]}\end{array}$ & $\begin{array}{c}\text { UTM-N } \\
{[\mathrm{m}]}\end{array}$ & $\begin{array}{c}\text { Altitud } \\
(\mathrm{m} \text { s.n.m) }\end{array}$ \\
\hline La Greda & 268300 & 6373900 & 25 \\
\hline Los Maitenes & 270075 & 6372161 & 25 \\
\hline Puchuncaví & 273800 & 6376700 & 35 \\
\hline Sur & 267434 & 6368062 & 25 \\
\hline Valle Alegre & 271800 & 6367420 & 20 \\
\hline
\end{tabular}

Tabla 2. Localización de las fuentes emisoras.

\begin{tabular}{|c|c|c|c|c|}
\hline \multicolumn{2}{|c|}{ Fuente } & $\begin{array}{c}\text { UTM-E } \\
{[\mathrm{m}]}\end{array}$ & $\begin{array}{c}\text { UTM-N } \\
{[\mathrm{m}]}\end{array}$ & $\begin{array}{c}\text { Altitud } \\
\text { (m s.n.m) }\end{array}$ \\
\hline Fundición Las Ventanas & Horno & 267780 & 6372680 & 18 \\
\hline & Planta de ácido & 267800 & 6372800 & 18 \\
\hline AES Gener S.A. & Unidad 1 & 267520 & 6373630 & 6 \\
\hline & Unidad 2 & 267490 & 6373700 & 6 \\
\hline
\end{tabular}


El análisis de los resultados correspondió a la comparación de los valores entregados por el modelo ISC3 y los valores filtrados, con los datos reales existentes para la red de receptores. Para ello, en primer lugar, se utilizaron los estimadores estadísticos recomendados por U.S EPA [14] que son: el promedio, la desviación estándar, la raíz del error cuadrático medio (RMSE), la diferencia media normalizada (NMD), el índice de acuerdo (IA). En segundo lugar, para evaluar las diferencias relativas entre el modelo y las observaciones se utilizaron dos nuevas herramientas estadísticas que son, el Factor normalizado medio del bias y el Factor de error medio normalizado [15], y que en comparación con las medidas tradicionales utilizadas como son la desviación media normalizada y el error relativo, tratan de manera simétrica los problemas relacionados con las sobre y subestimaciones presentadas por los modelos de calidad del aire.

\section{RESULTADOS Y DISCUSIÓN}

El presente trabajo entrega como resultados las concentraciones horarias y promedio de 24 horas en $\left[\mu \mathrm{g} / \mathrm{m}^{3}\right]$ de anhídrido sulfuroso predichas por el modelo ISC3 junto con las concentraciones filtradas entregadas por el filtro Kalman, en las cinco estaciones que conforman la red de calidad del aire del Complejo Industrial Las Ventanas; los resultados se obtuvieron representados gráficamente y en ellos se observan las concentraciones horarias y promedio de 24 horas modeladas, filtradas y observadas. En la figura 1 y figura 2 se presentan a modo de ejemplo las gráficas resultantes de la modelación horaria y promedio de 24 horas en la estación Los Maitenes.

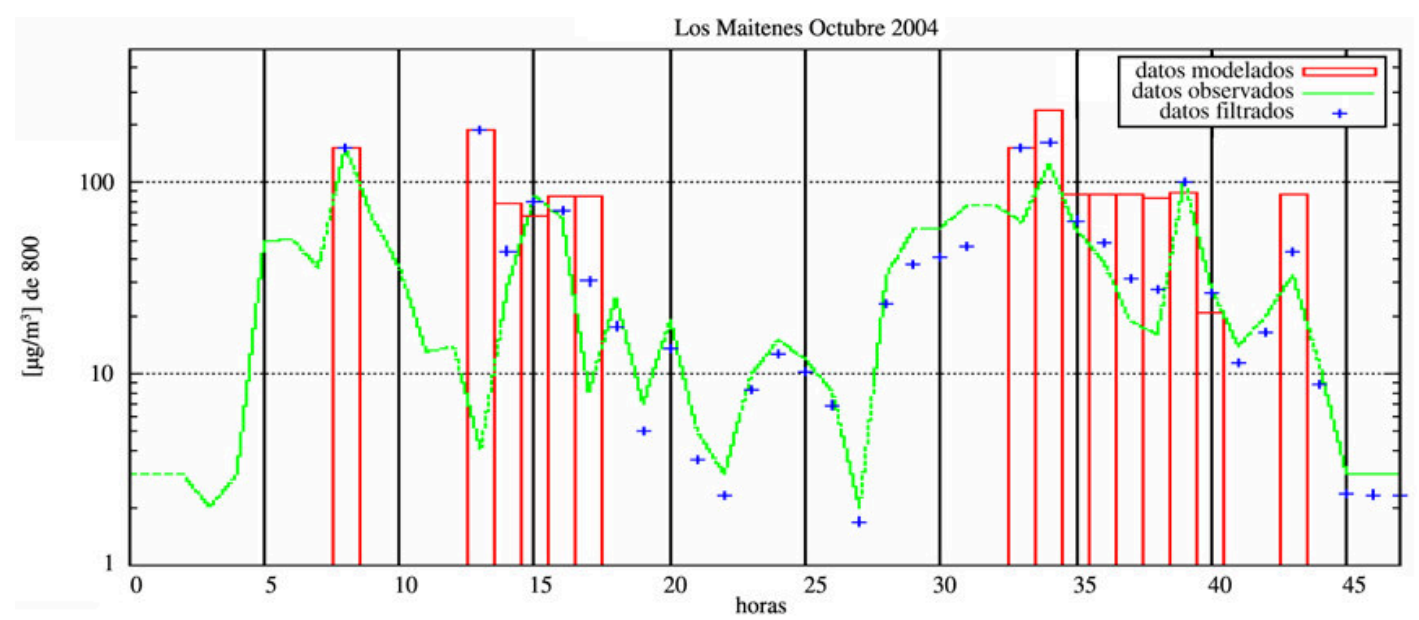

Figura 1. Concentraciones horarias observadas (verde), modeladas (rojo) y filtradas (azul) para octubre de 2004 en Los Maitenes.

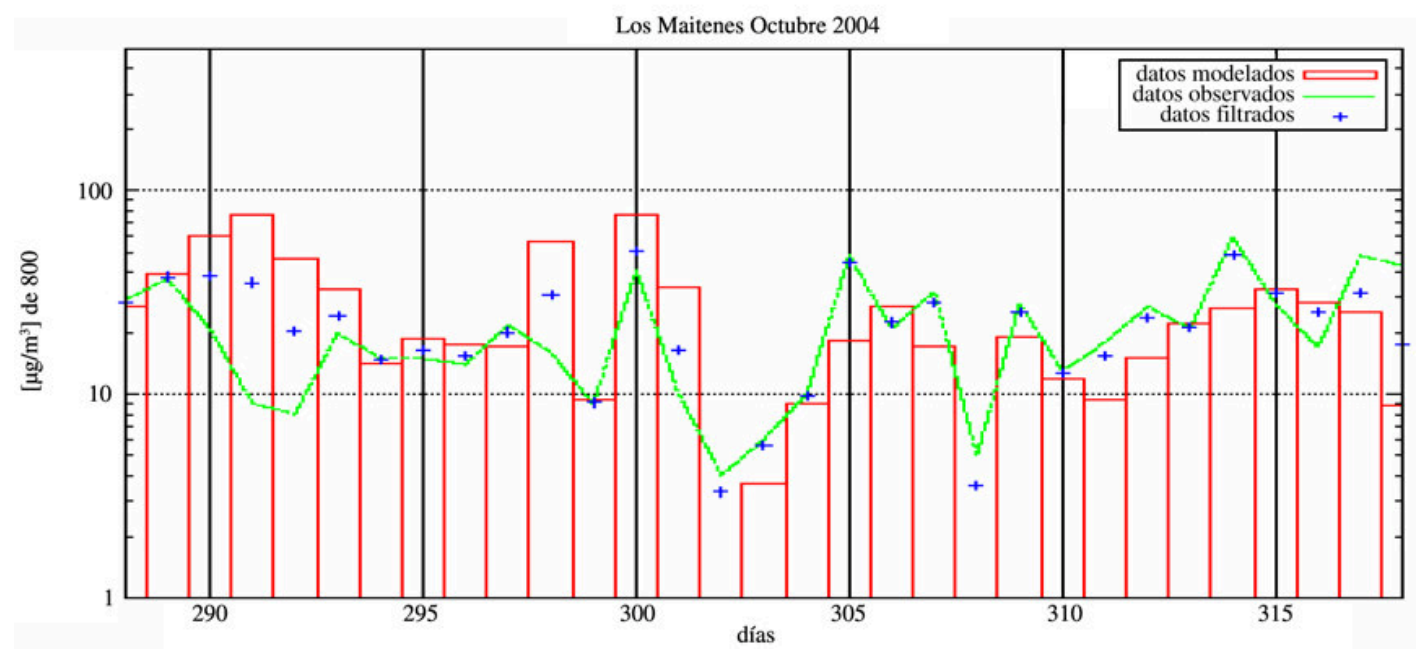

Figura 2. Concentraciones promedio de 24 horas observadas (verde), modeladas (rojo) y filtradas (azul) para octubre de 2004 en Los Maitenes. 


\section{Modelación de concentraciones horarias}

En la tabla 3 se observa que el modelo entrega el mejor desempeño en la estación Los Maitenes, con IA $\geq 0,50$ para los dos años analizados. Si bien se obtienen elevados valores de RMSE, para esta estación el modelo entrega la menor subestimación de las concentraciones observadas con $\mathrm{B}_{\mathrm{NMBF}}$ promedio de $-1,51$ para el año $2003 \mathrm{y}$ $-1,67$ para el 2004. Los valores de $\mathrm{E}_{\mathrm{NMEF}}$ también son menores. En cuanto a las concentraciones filtradas, se observa tal como era esperado que los errores diminuyen considerablemente alcanzando un $\mathrm{E}_{\mathrm{NMEF}}$ de 0,82 y 0,84 para los años 2003 y 2004 respectivamente. La tendencia del modelo a subestimar las concentraciones observadas también decrece alcanzando 1,40 en promedio para ambos años. Por otra parte, el IA mejora notoriamente con un valor promedio de 0,82 para ambos años.

Se observa que para las restantes cuatro estaciones los resultados obtenidos de la modelación horaria no son tan satisfactorios. En la estación La Greda el modelo mantiene la tendencia a subestimar las concentraciones observadas. La estación Valle Alegre presenta el menor valor RMSE, con un valor promedio de 10,6 para ambos años, sin embargo, el valor de IA es menor a 0,5. Para las concentraciones filtradas el IA alcanza valores aceptables con promedios anuales de 0,57 y 0,50 para el 2003 y 2004 respectivamente. En relación a La Greda, los valores de RMSE modelados para los años 2003 y 2004 alcanzan 77,85 y 82,44 respectivamente y a pesar de que para las concentraciones modeladas el $\mathrm{IA} \approx 0,27$, para las filtradas este valor se eleva a 0,62 en promedio para ambos años, con concentraciones que alcanzan valores promedio de 1,01 veces el valor de la observación. Para Puchuncaví y Sur ningún mes en ambas estaciones presenta un IA superior a 0,45 , encontrándose la mayor parte de los valores alrededor de 0,29 y 0,35 respectivamente. Por otro lado, se observan los más altos valores de $\mathrm{B}_{\mathrm{NMBF}}$ con máximos de $-15,47$ y $-14,31$ y $\mathrm{E}_{\mathrm{NMEF}}$ de 15,82 y 14,98 para los años 2003 y 2004 respectivamente, lo cual indica marcadas diferencias relativas con los valores observados. En cuanto a las concentraciones filtradas para ambas estaciones, aunque los errores se amortiguan no se logra en promedio un IA superior a 0,5 .

\section{Modelación de concentraciones promedio de 24 horas}

En la tabla 4 se observa un mejor desempeño del modelo ISC3 en todas las estaciones de calidad del aire, aunque la tendencia del modelo a subestimar las concentraciones observadas se mantiene. Del mismo modo que para la modelación horaria, se observa que el modelo presenta el mejor ajuste de concentraciones en la estación Los Maitenes con IA $\geq 0,5$, y a pesar de entregar RMSE elevados, presenta los menores valores de NMD y $\mathrm{E}_{\mathrm{NMEF}}$, llegando este último a alcanzar un valor promedio para los años 2003 y 2004 de 1,01 y 1,15 respectivamente, lo cual indica pequeñas diferencias relativas con los datos observados para esta estación. Por otra parte, esta estación continúa presentando la menor subestimación de las concentraciones observadas con un promedio anual no superior $\mathrm{a}-1,7$.

En cuanto a las concentraciones filtradas se observa que el IA es cercano a 0,80 para ambos años, los valores de NMD disminuyen en $36 \%$ y $43 \%$ y los de $\mathrm{E}_{\mathrm{NMEF}}$ en $23 \%$ y $26 \%$ para los años 2003 y 2004 respectivamente. Para las estaciones restantes, los valores de IA de las concentraciones modeladas no superan en promedio 0,5 para los periodos en estudio, salvo para La Greda que en el 2004 alcanza un IA de 0,56. Al igual que para la modelación horaria Valle Alegre presenta los menores valores de RMSE, con un valor de 6,82 en promedio para ambos años.

Tabla 3. Parámetros estadísticos calculados para las concentraciones horarias modeladas y filtradas para los años 2003 y 2004 en las cinco estaciones de calidad del aire.

\begin{tabular}{|c|c|c|c|c|c|c|c|c|c|c|c|}
\hline \multirow{2}{*}{\multicolumn{2}{|c|}{$\begin{array}{l}\text { Concentraciones } \\
\text { horarias }\left[\mu \mathrm{g} / \mathrm{m}^{3}\right]\end{array}$}} & \multicolumn{5}{|c|}{ Concentraciones Modeladas } & \multicolumn{5}{|c|}{ Concentraciones Filtradas } \\
\hline & & RMSE & NMD & IA & $\mathrm{B}_{\mathrm{NMBF}}$ & $\mathrm{E}_{\mathrm{NMEF}}$ & RMSE & NMD & IA & $\mathrm{B}_{\mathrm{NMBF}}$ & $\mathrm{E}_{\mathrm{NMEF}}$ \\
\hline \multirow{5}{*}{$\begin{array}{l}0 \\
3\end{array}$} & Los Maitenes & 83.59 & 27.36 & 0.51 & -0.51 & 1.68 & 54.98 & 24.91 & 0.82 & -0.36 & 0.82 \\
\hline & La Greda & 77.85 & -47.68 & 0.27 & 0.37 & 2.38 & 45.30 & 0.64 & 0.56 & -0.10 & 1.07 \\
\hline & Sur & 73.75 & 78.07 & 0.30 & -5.94 & 7.49 & 66.11 & 70.81 & 0.42 & -3.58 & 4.24 \\
\hline & Puchuncaví & 29.63 & 82.34 & 0.35 & -5.25 & 6.10 & 26.51 & 69.73 & 0.49 & -2.46 & 2.74 \\
\hline & Valle Alegre & 15.49 & 48.25 & 0.33 & -1.33 & 2.86 & 10.06 & 39.51 & 0.57 & -0.75 & 1.19 \\
\hline \multirow{5}{*}{$\begin{array}{l}2 \\
0 \\
0 \\
4\end{array}$} & Los Maitenes & 95.21 & 28.39 & 0.51 & -0.67 & 1.75 & 62.50 & 27.07 & 0.82 & -0.44 & 0.84 \\
\hline & La Greda & 82.44 & -41.68 & 0.28 & 0.30 & 2.33 & 46.10 & 1.27 & 0.68 & -0.08 & 0.97 \\
\hline & Sur & 65.81 & 68.50 & 0.29 & -4.35 & 5.90 & 58.13 & 67.79 & 0.43 & -2.94 & 3.70 \\
\hline & Puchuncaví & 43.46 & 85.23 & 0.35 & -7.47 & 8.29 & 39.81 & 73.02 & 0.46 & -3.42 & 3.69 \\
\hline & Valle Alegre & 15.62 & 42.81 & 0.31 & -1.30 & 3.00 & 11.23 & 34.41 & 0.50 & -0.67 & 1.32 \\
\hline
\end{tabular}


Tabla 4. Parámetros estadísticos calculados para las concentraciones promedio de 24 horas modeladas y filtradas para los años 2003 y 2004 en las cinco estaciones de calidad del aire.

\begin{tabular}{|c|c|c|c|c|c|c|c|c|c|c|c|}
\hline \multirow{2}{*}{\multicolumn{2}{|c|}{$\begin{array}{c}\text { Concentraciones } \\
\text { Promedio de } 24 \\
\text { horas }\left[\mu \mathrm{g} / \mathrm{m}^{3}\right]\end{array}$}} & \multicolumn{5}{|c|}{ Concentraciones Modeladas } & \multicolumn{5}{|c|}{ Concentraciones Filtradas } \\
\hline & & RMSE & NMD & IA & $\mathrm{B}_{\mathrm{NMBF}}$ & $\mathrm{E}_{\mathrm{NMEF}}$ & RMSE & NMD & IA & $\mathrm{B}_{\mathrm{NMBF}}$ & $\mathrm{E}_{\mathrm{NMEF}}$ \\
\hline \multirow{5}{*}{$\begin{array}{l}2 \\
0 \\
0 \\
3\end{array}$} & Los Maitenes & 28,27 & 30,02 & 0,56 & $-0,56$ & 1,01 & 21.27 & 23.04 & 0.75 & -0.36 & 0.65 \\
\hline & La Greda & 23,16 & $-42,51$ & 0,49 & 0,32 & 1,45 & 7.79 & 1.05 & 0.94 & -0.03 & 0.29 \\
\hline & Sur & 38,44 & 78,69 & 0,41 & $-5,74$ & 6,09 & 32.04 & 62.13 & 0.56 & -2.85 & 3.00 \\
\hline & Puchuncaví & 20,45 & 84,50 & 0,44 & $-6,25$ & 6,35 & 19.31 & 78.43 & 0.48 & -4.42 & 4.48 \\
\hline & Valle Alegre & 6,83 & 49,27 & 0,47 & $-1,32$ & 1,74 & 3.91 & 27.32 & 0.77 & -0.46 & 0.60 \\
\hline \multirow{5}{*}{$\begin{array}{l}2 \\
0 \\
0 \\
4\end{array}$} & Los Maitenes & 33,05 & 27,94 & 0,52 & $-0,66$ & 1,15 & 22.63 & 20.67 & 0.79 & -0.38 & 0.66 \\
\hline & La Greda & 24,29 & $-40,20$ & 0,56 & 0,29 & 1,40 & 10.51 & -6.62 & 0.89 & 0.03 & 0.45 \\
\hline & Sur & 33,46 & 68,16 & 0,43 & $-4,35$ & 4,76 & 28.82 & 55.93 & 0.56 & -2.55 & 2.78 \\
\hline & Puchuncaví & 26,97 & 85,27 & 0,44 & $-7,54$ & 7,57 & 25.77 & 79.29 & 0.48 & -6.00 & 6.02 \\
\hline & Valle Alegre & 6,82 & 41,26 & 0,41 & $-1,27$ & 2,00 & 3.31 & 20.48 & 0.82 & -0.36 & 1.39 \\
\hline
\end{tabular}

Es importante mencionar que los resultados obtenidos referentes a la modelación de concentraciones promedio están de acuerdo con un trabajo anteriormente realizado en la zona de Ventanas en el cual se evalúa el desempeño de tres modelos de dispersión atmosférica [16], uno de los cuales es ISC3 en función de las estaciones de calidad del aire Valle Alegre, La Greda y Puchuncaví. Si bien sólo se analizan tres estaciones de monitoreo los resultados obtenidos son del mismo orden de magnitud que en el presente trabajo.

En cuanto a las concentraciones filtradas en general, las estaciones Sur, Valle Alegre y La Greda entregan IA superiores a 0,6. Para Puchuncaví el IA no es superior a 0,5 con un promedio de 0,48 para ambos años.

En un análisis general de los resultados filtrados se observa que La Greda entrega mejores resultados que Los Maitenes con $I A \approx 0,9$; la tendencia a subestimar las concentraciones se mantiene, aunque es mucho menor; por otra parte, los valores de $\mathrm{E}_{\mathrm{NMEF}}$ también son menores para esta estación, lo cual indica leves diferencias relativas entre las concentraciones filtradas y observadas.

Considerando que existen ciertos factores que pueden influir en la calidad de los resultados de la modelación, los cuales son: la tasa de emisión del contaminante utilizado y los datos meteorológicos, se realizó un análisis de sensibilidad frente a cada uno de estos factores en forma independiente, el cual es presentado a continuación.

\section{Análisis de sensibilidad frente a la variación en las emisiones}

Debido a que la modelación entregó subestimaciones de las concentraciones reales, se realizó una modelación de las concentraciones horarias de $\mathrm{SO}_{2}$ considerando sólo el aumento de las emisiones producidas por las fuentes consideradas, incrementando éstas un $12,5 \%$ hasta doblar las emisiones originales. Esta modelación se llevó a cabo en las estaciones que entregaron el menor y mayor valor de RMSE para los años 2003-2004, realizando ambas modelaciones para el mes que entrega el mayor error.

De este análisis se desprende que el IA en la estación de Los Maitenes presenta un leve y sostenido aumento de aproximadamente 0,01 por cada incremento del $12,5 \%$ en la tasa de emisión, por lo cual y considerando que se mantiene esta tendencia lineal se logrará un ajuste aceptable $(\mathrm{IA}=0,5)$ para un aumento del $310 \%$ sobre las emisiones inicialmente consideradas. Un comportamiento inverso presenta la estación Valle Alegre, para la cual el IA disminuye en igual proporción.

En general, se observa que el IA presenta un aumento o disminución del $4 \%$ por cada $12,5 \%$ de incremento de emisión.

\section{Análisis de sensibilidad frente a la variación de la velocidad del viento}

Este análisis comprendió la realización de una modelación horaria en las cinco estaciones de calidad del aire para el mes de enero de 2004. En la tabla 5 se presentan los rangos de velocidad del viento considerados para el análisis.

Tabla 5. Rangos de velocidad del viento.

\begin{tabular}{|c|c|c|c|}
\hline \multicolumn{4}{|c|}{ Rangos de velocidad del viento en $[\mathrm{m} / \mathrm{s}]$} \\
\hline$v<2$ & $2 \leq v<4$ & $4 \leq v<6$ & $6 \geq v$ \\
\hline
\end{tabular}


A partir de esta análisis se observa que las estaciones Sur y La Greda presentan un mejor desempeño para velocidades del viento de 4 a $6[\mathrm{~m} / \mathrm{s}]$, del mismo modo, para Valle Alegre entrega el mejor acuerdo para velocidades mayores a $6[\mathrm{~m} / \mathrm{s}]$. Esto en parte explica los altos errores obtenidos para estas estaciones debido a que la mayor parte de las mediciones horarias de velocidad del viento en la estación Principal presenta valores menores a este rango. Los Maitenes entrega mejores resultados velocidades del viento menores a $4[\mathrm{~m} / \mathrm{s}]$, lo cual confirma el mejor desempeño del modelo para esta última estación. En el caso de Puchuncaví se logra un mejor desempeño para velocidades entre 0 y $4[\mathrm{~m} / \mathrm{s}]$.

\section{Análisis de sensibilidad frente a la variación de dirección de los vientos}

Este análisis corresponde a una modelación de concentraciones horarias frente a las distintas direcciones de viento en la estación Los Maitenes.

De este análisis se obtiene que el modelo presenta un mejor desempeño del modelo para las direcciones Este-Oeste y Noroeste-Sureste, observándose para las últimas direcciones un mejor ajuste entre las concentraciones observadas y modeladas. Se observan IA $\geq 0,5$ para las direcciones en la línea Este-Oeste, Nor-noroeste con Sur-sureste, Noroeste con Sureste, Oeste-noroeste con Este-sureste y Oeste-suroeste con Este-noreste, presentándose los más altos valores para las dos primeras direcciones con un valor de 0,75 y 0,68 respectivamente.

En general, se puede observar que el modelo ISC3 presenta un mejor desempeño en la dirección Este-Oeste, que es donde se presenta el mayor porcentaje de incidencia de los vientos y donde se localiza la estación Los Maitenes, la cual entrega los mejores resultados tanto para la modelación horaria como del promedio de 24 horas. Si bien en las direcciones Noroeste-Sureste y Nor-noroeste con Sur-sureste se obtiene un alto valor de IA, el error obtenido para la dirección Noroeste con Sureste indica concentraciones modeladas que casi duplican el valor de las reales, por otro lado, el porcentaje de incidencia de vientos en estas direcciones es bajo, lo cual explica que el resultado de la modelación en la estación Valle Alegre ubicada entre éstas no sea el mejor. Para las direcciones en la línea Norte-Sur, Nor-noreste con Sur-suroeste y Noreste con Suroeste, que son en las que se localizan las estaciones La Greda, Sur y Puchuncaví, los valores de IA no son aceptables, lo que coincide con la baja incidencia de vientos en estas direcciones y explica el bajo desempeño del modelo en estas tres estaciones.

\section{CONCLUSIONES}

Del presente trabajo se concluye que el modelo ISC3 muestra una tendencia a subestimar las concentraciones modeladas de anhídrido sulfuroso en las cinco estaciones de calidad del aire, siendo ésta más marcada en las estaciones Sur y Puchuncaví, tendencia que se presenta tanto para la modelación de concentraciones horarias como de promedios de 24 horas. Para la modelación de ambas concentraciones la estación que presenta mejores resultados es Los Maitenes y aunque los valores de raíz del error cuadrático medio (RMSE) son los más altos dentro de las estaciones en estudio, se observan índices de acuerdo (IA) superiores a 0,5 ; la menor subestimación de las concentraciones observadas y menores diferencias relativas en comparación con las cuatro estaciones restantes. En general, el modelo ISC3 entrega un desempeño aceptable (IA $\geq 0,5$ ) en la estación Los Maitenes para los años 2003 y 2004, como también, muy buen ajuste para algunos meses en La Greda y Valle Alegre, en la modelación de las concentraciones horarias de anhídrido sulfuroso. En cuanto a la modelación de concentraciones promedio de 24 horas, el modelo ISC 3 es capaz de predecir concentraciones de anhídrido sulfuroso con menor error y mayor índice de acuerdo (IA) para todas las estaciones, presentando un mejor desempeño en las estaciones Los Maitenes, La Greda y Valle Alegre de las cinco estaciones consideradas. El hecho de que la estación Los Maitenes se encuentre localizada en la dirección en que los vientos soplan con mayor frecuencia, es uno de los principales factores que influyen en el mejor desempeño que el modelo presenta para esta estación. Otro factor que influye en el menor desempeño del modelo en tres de las cinco estaciones, está en relación directa con la velocidad de los vientos, debido a que en las estaciones Sur, La Greda y Valle Alegre el modelo entrega mejores resultados para velocidades mayores a $4[\mathrm{~m} / \mathrm{s}]$, rango de velocidades de viento menos predominantes dentro de los datos meteorológicos medidos en la estación Principal.

Por su parte, el empleo del filtro Kalman mejora en gran medida las concentraciones modeladas, minimizando el error y aumentando el ajuste de los datos, obteniendo elevados valores de IA en tres de las cinco estaciones de calidad del aire para la modelación horaria, y para todas las estaciones en la modelación de concentraciones promedio.

En resumen, el modelo ISC3 predice con menor error concentraciones promedio de 24 horas que concentraciones horarias, siendo mucho más aplicable en la modelación de concentraciones en puntos localizados en la dirección predominante de los vientos, que en la obtención de 
una visión global de las concentraciones de anhídrido sulfuroso en la comuna de Puchuncaví.

\section{REFERENCIAS}

[1] C. Mena. "Análisis de la situación ambiental en la zona de Puchuncaví producida por la minería y sus perspectivas". Tesis para optar al título de Ingeniero Industrial. Universidad Técnica Federico Santa María. 1996.

[2] D.S. $N^{0}$ 185. Decreto que regula a los establecimientos y fuentes emisoras de anhídrido sulfuroso, material particulado o arsénico.

[3] X. Doménech. "Química Atmosférica: Origen y efectos de la contaminación”. Ediciones Miraguano. 1995.

[4] E. Derek. "La Contaminación Atmosférica". Editorial Cátedra. 1990

[5] Pacific Environmental Services. "Users Guide for the Industrial Source Complex (ISC3) Dispersion Models". Volumen I: Instrucciones para el usuario. Agencia de protección ambiental de EE.UU. (EPA). 1995.

[6] Pacific Environmental Services. "Users Guide for the Industrial Source Complex (ISC3) Dispersion Models". Volumen II: Descripción de los algoritmos del modelo. Agencia de protección ambiental de EE.UU. (EPA). 1995.

[7] G. Welch and G. Bishop. "An Introduction to the Kalman filter". University of North Carolina at Chapel Hill. 2004. http://vision.ee.pusan.ac.kr/
[8] A. Solera. "El Filtro de Kalman". Banco Central de Costa Rica. 2003. www.bccr.fi.cr

[9] Instituto Nacional de Estadísticas. www.ine.cl.

[10] M. Silva. "Análisis del impacto de las emisiones de $\mathrm{SO}_{2}$ en el Valle de Aconcagua, Sector La Calera". Tesis para optar al título de Ingeniero Civil Químico. Universidad Técnica Federico Santa María. 2003.

[11] CONAMA. "Evaluación del plan de descontaminación de Ventanas”. Junio 2003. www.conama.cl

[12] D. Sevilla. "Programación en Perl: Programación para Internet: Perl \& CGI”. 1999.

[13] “Introducción al Perl”. Centro de Innovación para la Sociedad de la Información. Universidad de Las Palmas de Gran Canaria. 2005. www.cicei.com

[14] K. Gronskei, F. Gram, S. Larssen and S. Walker. "Evaluation of urban scale time-dependent dispersion model with subgrid elements in Oslo, Norway". Norsk institutt for luftforskning. 2001. www.nilu.no

[15] Shaocai Yu, Brian Eder, Robin Dennis, ShaoHang Chu and Stephen Schwartz. "New unbiased symmetric metrics for evaluation of the air quality models". Office of air quality planning and standards. U.S. EPA. www.cmascenter.org

[16] N. Barraza. "Modelos de Dispersión de Contaminantes atmosféricos: comparación de desempeños en zona de Ventanas, V Región”. 1998. www2.ing.puc.cl 